\title{
Using Neuroscience to Broaden Emotion Regulation: Theoretical and Methodological Considerations
}

\author{
Elliot T. Berkman* and Matthew D. Lieberman \\ University of California, Los Angeles
}

\begin{abstract}
Behavioral research on emotion regulation thus far has focused on conscious and deliberative strategies such as reappraisal. Neuroscience investigations into emotion regulation have followed suit. However, neuroimaging tools now open the door to investigate more automatic forms of emotion regulation that take place incidentally and potentially outside of participant awareness that have previously been difficult to examine. The present paper reviews studies on the neuroscience of intentional/deliberate emotion regulation and identifies opportunities for future directions that have not yet been addressed. The authors suggest a broad framework for emotion regulation that includes both deliberative and incidental forms. This framework allows insights from incidental emotion regulation to address open questions about existing work, and vice versa. Several studies relevant to incidental emotion regulation are reviewed with the goal of providing an empirical and methodological groundwork for future research. Finally, several theoretical issues for incidental and intentional emotion regulation are discussed.
\end{abstract}

\section{Using Neuroscience to Broaden Emotion Regulation: Theoretical and Methodological Considerations}

The study of emotion regulation - the set of processes that alter emotional experience - has become a major area of research for scientists seeking a complete picture of human emotional experience. Researchers have made progress by clearly documenting the powerful ability that people have to regulate their emotions (Gross, 2007) and elucidating the specific cognitive strategies they employ in order to do so. The advent of neuroimaging techniques such as functional magnetic resonance imaging (fMRI) has opened new avenues of inquiry into emotion regulation, beginning with the identification of the network of neural systems involved. Following in this line, further work has started to examine the time-course of regulation, as well as the complex interaction among systems typically implicated in emotion, cognitive control, and language. 
Though emotion regulation can include automatic processes (Gross \& Thompson, 2007), the majority of research focuses on types of emotion regulation that are effortful and intentional. This approach has provided the field with a broad theoretical foundation and empirical insight into the affective, cognitive, and physiological consequences of emotion regulation. One important direction for future research is to further investigate less deliberative, more incidental forms of emotion regulation. Below, we review several studies that highlight this kind of incidental or unintended emotion regulation. When considered in context with existing theory and data on emotion regulation, these studies suggest exciting and novel avenues of research.

A key reason why incidental forms of emotion regulation have not yet been explored is because participants are not necessarily aware that the regulation is taking place and thus cannot report on it. With the advent of neuroimaging, participants need not intend to regulate their emotions or even be aware of changes in their own experience in order to provide useful data. It is now possible to study incidental emotion regulation building upon existing theories of emotion regulation and the expanding neuroscientific literature on the neural systems involved in deliberative emotion regulation. Given that participants will have little to no awareness when emotion regulation takes place incidentally, neuroscience methods will be valuable in moving forward because they can measure ongoing neural processes without interrupting the psychological processes. Examining how these neural mechanisms vary across conditions can yield valuable insights into the dynamic psychological processes involved in incidental emotion regulation. However, neuroscience is also only beginning to address incidental mental phenomena and thus many conclusions drawn using these techniques are tentative. Nonetheless, neuroscientists are developing tools that can be used to address incidental effects (e.g. inferring mental states from neural activity; c.f. Poldrack, 2006), and these tools will continue to develop alongside with research on incidental emotion regulation.

In order to compare and contrast the two types of emotion regulation, we will provide a review of the existing data on the neuroscience of deliberative emotion regulation before turning to incidental emotion regulation. We will use the term intentional emotion regulation to refer to more deliberative and effortful attempts to control or alter one's own emotion experience. In contrast, incidental emotion regulation will be used to refer to more unintentional, non-conscious or automatic forms of emotion regulation, and to the unintended regulatory effects (e.g. reduced emotional intensity) of unrelated deliberate/controlled processes (e.g. a motor task). Though we draw a distinction between intentional and incidental forms of regulation for comparison, we view all forms of emotion regulation as falling on a continuum (Gross \& Thompson, 2007). For example, it might require a great deal of effort for a taxi driver in New 
York to regulate his frustration in traffic at first, but with time and practice, that same frustration might come to be regulated with less effort and perhaps even without intention.

\section{Review of Neuroscience Investigations of Emotion Regulation}

Much of the current neuroscience literature on emotion regulation is based on the conceptual model articulated by Gross (1998a; 1998b; 1999), shown in Figure 1. Various emotion regulation strategies are differentiated by where they occur on a timeline. Emotion regulation strategies that occur earlier in time, following exposure to some emotion-provoking external stimulus but before a fully developed emotional response (including but not limited to changes in facial expression, subjective experience, cognitive appraisal, and peripheral physiological changes; see Ekman, 1992) are termed 'antecedent-focused' strategies. Because of their timing relative to an emotional response, these strategies typically involve changing the external stimulus in some way in order to prevent what otherwise would become a full emotional response. The stimulus can be changed conceptually by reinterpreting the meaning of the stimulus ('reappraisal') or changed de facto by altering the situation (e.g. leaving the room). These types of strategies are relatively proactive because they can take place near the beginning of the emotion time course or even before an emotional event begins.

In contrast, strategies that take place later in time are known as 'response-focused' strategies because of their focus on altering the emotional response after it has occurred. The paradigmatic example of a response-focused strategy is 'behavioral suppression', which involves

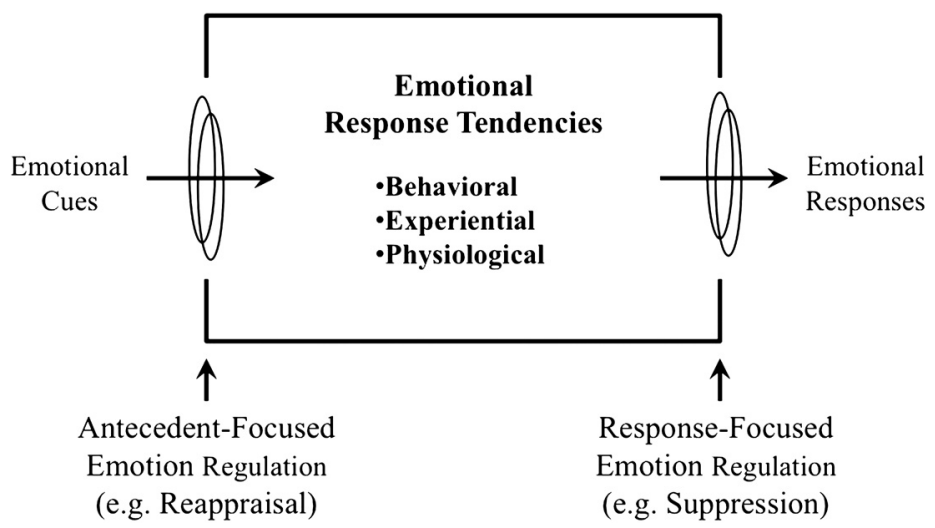

Figure 1 The dominant process model of intentional emotion regulation. Adapted (with permission) from Gross, 1998a. 
inhibiting external displays of emotion such as the facial expression. These kinds of emotion regulation strategies are essentially reactive, being recruited to alter the emotional response only after it has begun. As the name suggests, the emphasis of response-focused strategies is on altering one or more components of the emotional response rather than the eliciting stimulus.

Neuroimaging studies following the Gross model typically present participants in an fMRI scanner with emotionally evocative and neutral stimuli, then compare across conditions when participants either engage in some form of emotion regulation (e.g. antecedent- vs. response-focused) or simply view the stimuli. For example, Ochsner, Bunge, Gross, and Gabrieli (2002) compared neural activation between reappraisal (an antecedent-focused exemplar) to passive viewing trials. Compared to passive viewing, reappraisal recruited broad regions of the left lateral prefrontal cortex (LPFC), dorsomedial prefrontal cortex (DMPFC), temporal pole, and a single region in the right hemisphere, the supramarginal gyrus. The authors note that regions in this network have been implicated in processes such as top-down, effortful control, particularly involving language (left LPFC), attention to emotional states (DMPFC), and the integration of visuospatial location or rotation with linguistic information (supramarginal gyrus). Participants' self-reported emotion regulation success (as measured by a reduction in negative affect) was associated with increased activation in the dorsal anterior cingulate cortex (dACC), a region frequently implicated in conflict monitoring during tasks that require the control of a prepotent response. Finally, reappraisal-related increases in left ventrolateral prefrontal cortex (VLPFC) were negatively correlated across subjects with regions implicated in evaluating affective salience such as the amygdala and medial orbital prefrontal cortex. Several other studies replicate the basic finding of increased LPFC, VLPFC, DMPFC, and dACC (though not all with the left-lateralization in the prefrontal cortex) and decreased amygdala and MOFC during reappraisal (Kim \& Hamann, 2007; Ochsner et al., 2004; Phan et al., 2005).

Other studies that examine different cognitive strategies can help distinguish those neural systems that are unique to reappraisal from those that are involved in emotion regulation per se. Beauregard, Levesque, and Bourgouin (2001) instructed their participants to use 'cognitive distancing' to regulate their responses to erotic videos ('distance yourself from these stimuli . . to become a detached observer'). This emotion regulation strategy and reappraisal are both primarily linguistic in nature. However, unlike reappraisal, in cognitive distancing participants alter their relationship with the scene (i.e. 1st vs. 3rd person) rather than their interpretation of the events depicted therein. Compared to attending to neutral films, engaging in cognitive distancing recruited activity in bilateral LPFC and ACC. Furthermore, the activation in the amygdala and temporal poles that had been observed in the attend condition (compared to attending 
to neutral films) was not observed in the distancing condition (compared to attending to neutral films), suggesting that these regions may have been down-regulated by distancing. A subsequent study by the same group also found right orbital and dorsal LPFC to be involved in cognitive distancing from sad films (Levesque et al., 2003).

In another study comparing among emotion regulation strategies, Goldin, McRae, Ramel, and Gross (2008) compared cognitive reappraisal to behavioral suppression of affect, and found a similar network of regions for both strategies including bilateral VLPFC and right DLPFC. The key differences between reappraisal and suppression in their study were (1) the time course of the strategies, with the neural correlates of reappraisal occurring earlier ( $0-4.5$ seconds following stimulus onset) and the neural correlates of suppression occurring later ( $>10$ seconds following onset), and (2) the concomitant effects on emotion regions, with reappraisal resulting in reductions in amygdala and insula, and suppression resulting in no change or increases in those structures.

Table 1 summarizes the findings from 20 studies on deliberate forms of emotion regulation, some of which were reviewed here. These studies suggest that the prefrontal cortex is broadly involved in deliberative forms of emotion regulation, and especially the ventrolateral aspects of the prefrontal cortex, bilaterally. Additionally, about half of these studies find activity in the posterior portion of the dorsomedial prefrontal cortex/ superior frontal gyrus (BA 6/8). A few studies also find activation in the dorsolateral prefrontal cortex bilaterally, and there is inconsistent support for more posterior regions such as the posterior temporal gyrii and inferior parietal lobules.

\section{Broadening Emotion Regulation}

Tremendous progress has been made toward building a neuroscience of emotion regulation over the past few years. Still, there are a number of opportunities to build upon the literature and address important unanswered questions. We will briefly summarize what we consider to be the most interesting of these and suggest ways in which they might be addressed in future studies.

Do other operationalizations of regulation elicit the same pattern of neural activity?

Most studies use one of several operationalizations of emotion regulation (e.g. reappraisal, distraction or distancing) and draw conclusions based on comparisons of that condition to an 'attend' or other control condition. Unless the goal of a study is to develop new operationalizations of emotion regulation, most researchers tend to use operationalizations that have worked in the past. As a consequence, nearly all of the studies in the emotion regulation literature to date investigate the same handful of 
Table 1 Summary of results from neuroimaging studies on intentional emotion regulation

\begin{tabular}{|c|c|c|c|c|c|c|c|c|c|c|c|c|}
\hline \multirow[b]{3}{*}{ Study } & \multirow[b]{3}{*}{ First author } & \multirow[b]{3}{*}{ Year } & \multirow[b]{3}{*}{ Contrast } & \multicolumn{7}{|c|}{ Prefrontal cortex } & \multicolumn{2}{|c|}{ ACC } \\
\hline & & & & \multicolumn{2}{|c|}{ Ventrolateral } & \multicolumn{2}{|c|}{ Dorsolateral } & \multirow{2}{*}{$\begin{array}{l}\text { Lateral } \\
\text { BA6/8 } \\
\text { dorsal }\end{array}$} & \multicolumn{2}{|c|}{ Medial } & \multirow[b]{2}{*}{ Rostral } & \multirow[b]{2}{*}{ Dorsa } \\
\hline & & & & Right & Left & Right & Left & & Dorsal & Medial & & \\
\hline 1 & Banks & 2007 & Reappraise > Maintain & - & - & - & - & & - & & & \\
\hline 2 & Beauregard & 2001 & Reappraise $>$ View & $\bullet$ & & & & & & & - & \\
\hline 3 & Eippert & 2007 & Reappraise $>$ View & & - & & - & & & & & - \\
\hline $\begin{array}{l}4 \\
5\end{array}$ & Goldin & 2008 & $\begin{array}{l}\text { Suppress > View } \\
\text { Reappraise }>\text { View }\end{array}$ & $\bullet$ & $\bullet$ & $\bullet$ & & $\bullet$ & & & & \\
\hline $\begin{array}{l}3 \\
6\end{array}$ & Harenski & 2006 & $\begin{array}{l}\text { Reappraise > View } \\
\text { Reappraise }>\text { View }\end{array}$ & $\bullet$ & $\bullet$ & & & - & & - & & \\
\hline 7 & Herwig & 2007 & Reappraise > View & & & & - & - & & & & \\
\hline 8 & Kalisch & 2005 & Detach > Identify & - & & & & & & & & \\
\hline 9 & Kalisch & 2006 & Self-distraction > View & & & & - & & & & & \\
\hline 10 & Kim & 2007 & Reappraise > View & - & - & & & - & - & & & - \\
\hline 11 & Lee & 2008 & Suppress > React & $\bullet$ & & & & $\bullet$ & & & & \\
\hline 12 & Levesque & 2003 & Detach $>$ View & $\bullet$ & & - & & & & & & \\
\hline 13 & McRae & 2008 & Reappraise > View & - & - & & & - & & & & \\
\hline 14 & Ochsner & 2002 & Reappraise > View & & - & & & - & & & & \\
\hline 15 & Ochsner & 2004 & Reappraise > View & - & - & - & - & - & & & & \\
\hline 16 & Ohira & 2006 & Suppress > View & & - & & & & & & & \\
\hline 17 & Phan & 2005 & Reappriase > Maintain & - & & & & & - & & & $\bullet$ \\
\hline 18 & Schaefer & 2003 & $\begin{array}{l}\text { Propositional reappraisal > } \\
\text { Schematic engagement }\end{array}$ & $\bullet$ & $\bullet$ & & & & & & & \\
\hline & Urry & 2006 & Reappraise $>$ View & & & & & & & & & \\
\hline \multirow[t]{2}{*}{20} & Wager & 2008 & Reappraise > View & $\bullet$ & $\bullet$ & $\bullet$ & $\bullet$ & $\bullet$ & $\bullet$ & & & \\
\hline & & & Number of studies & 14 & 12 & 5 & 6 & 9 & 4 & 1 & 1 & 3 \\
\hline
\end{tabular}

Note. ACC, anterior cingulate cortex; BA, Brodmann's Area. Numbers listed at the bottom represent a tally (out of 20) of studies showing activation in each region. 
operationalizations of emotion regulation (reappraisal, suppression, and distancing). Because few studies directly compare among multiple emotion regulation strategies (cf. Goldin et al., 2008), it is difficult to disentangle the neural systems involved in emotion regulation in general from those unique to a particular strategy.

Why should we care about other regulation strategies? Perhaps the best reason is that the construct of 'emotion regulation' encompasses a broad range of interesting psychological dimensions that have not yet been explored. In theory, regulation strategies can vary in their temporal location (relative to the stimulus onset), cognitive abstraction, effort, accessibility, and level of awareness, all dimensions that are likely to be important to the psychological experience and physiological outcomes of regulation. With only a small number of regulation strategies to work with, it is difficult to make conclusions about any one of these dimensions. For example, a study comparing reappraisal to suppression cannot make strong claims about antecedent- and response-focused strategies more broadly because reappraisal and suppression vary on a number of dimensions besides early-late. Reappraisal is more cognitive and suppression more behavioral, and reappraisal requires attention to internal psychological states such as beliefs whereas suppression requires attention to external physical states such as facial muscles. The 'specific strategies' approach that has been popular in the field has served us well in the past to answer important questions about the neural systems involved in emotion regulation. Moving forward to answer other questions will require new operationalizations of emotion regulation that are targeted at specific theoretical issues.

One way to achieve theoretical specificity is to manipulate regulation strategies along a single dimension. Neural regions that are recruited across a variety of strategies could be considered general to emotion regulation, and those that are sensitive to small changes in the instructions might be considered strategy-specific. In a recent example of this approach, Ochsner et al. (2004) compared the neural activation associated with two reappraisal strategies that differed only in the focus on selfvs. other-relevant information. In a 'conjunction' analysis of both types of reappraisal together, they observed increases in bilateral dorsal, ventral, and dorsomedial prefrontal cortices, suggesting that these regions are relatively independent of reappraisal strategy. In contrast, direct comparisons between self- and other-focused strategies showed relative increases in medial prefrontal regions for self-focused reappraisal, and relative increases in lateral prefrontal regions for other-focused reappraisal. Together with operationalizations that are targeted at specific theoretical constructs (e.g. extent of self-involvement in regulation), these analytic strategies can address important unanswered questions about how the neural systems involved in emotion regulation are modulated, or not, by any number of variables. 


\section{What can neuroscience studies tell us about psychological processes?}

It is tempting to infer which mental processes are involved in a task based on the brain regions that increase with the task. For example, nearly all of the studies cited thus far conclude that emotion regulation involves higher-order 'executive control' based on observed increases in DLPFC a region putatively involved in executive control - during regulation trials. Though appealing, this conclusion is not justified based solely on this data because the DLPFC, like nearly all other regions, is known to be involved in many other processes. For instance, the DLPFC may be involved in executive control, but it is also involved in spatial working memory (Casey et al., 1998), consequential foresight (Blakemore, Rees, \& Frith, 1998), and awareness of visual verbal stimuli (Kjaer, Nowak, Kjaer, Lou, \& Lou, 2001). Though it may well be the case that DLPFC is engaged in top-down control during these emotion regulation tasks, they only demonstrate that emotion regulation recruits the DLPFC.

Concluding that a specific mental process is occurring during a task because a particular brain region is relatively more active during that task is known as 'reverse inference' (Poldrack, 2006). The reverse inference problem focuses the need to more carefully specify and isolate the processes involved in experimental tasks used to study emotion regulation. Often, the comparisons used are not 'process-pure', meaning that they do not isolate a single mental process, thereby complicating the interpretation of brain activation changes to that task. For example, it is noted above that there are multiple differences between reappraisal and suppression, any of which might be driving the differences in activation. One way to address the reverse inference problem is to use parametric modulation analyses, in which a comparison is made across instances of the same task that systematically vary on one dimension. This approach is useful when it is difficult to manipulate a mental process across task conditions as is the case in comparing emotion regulation strategies. A simple example would be to have participants mentally vary their temporal distance from the same emotionally charged event (e.g. 1 minute vs. 1 week vs. 1 year following) instead of comparing a single distancing condition to an attend condition.

\section{Are emotion regulation capacity and tendency distinct?}

Most fMRI studies of emotion regulation are tightly controlled in that subjects receive extensive training in a particular emotion regulation strategy prior to scanning and subjects are given cues throughout the study indicating when they should and should not be regulating their emotional responses. Such procedures are essential to ensure that the desired emotion regulation processes are occurring at specific points in time so that the analyses will reflect the actual neural correlates of a specific form of emotion regulation. 
Assuming that motivation to follow experimental instructions is relatively high and similar across subjects, these studies can be characterized as assessing the capacity of subjects to perform emotion regulation tasks. All the subjects are presumably trying to perform the tasks, and thus, those who succeed presumably have a higher capacity for emotion regulation than those who fail. While poor emotion regulation capacity is surely one reason why some individuals are poor regulators in everyday life, it is not the only reason.

Many individuals who have high emotion regulation capacity may still be poor regulators in daily life because they lack the tendency to regulate their emotions. In other words, when there is no experimenter instructing subjects when to regulate, some with high capacity may not realize that they would benefit from regulating their emotions in certain circumstances or may not choose to put it the effort. Thus, it would seem critical to assess both the capacity and the tendency to regulate.

As it currently stands, there have been no fMRI investigations of emotion regulation tendency, as distinct from capacity (though there has been an investigation on real-world tendency; Gross, Richards, \& John, 2006). Such studies present methodological difficulties because even just asking subjects about their emotional experience after each of several trials might contaminate the natural response of the subject. To the extent that the presence of neural patterns uniquely associated with emotion regulation are present in emotional situations when emotion regulation is not explicitly required, this might reflect the tendency of the individual to spontaneously engage in emotion regulation (Eisenberger, Lieberman, \& Williams, 2003; Tabibnia et al., 2008). This is the same logic that several fMRI studies of incidental emotion regulation use for assessment, though this should not be taken to imply that spontaneous emotion regulation measured in 'tendency' assessments are not intentional or effortful.

Below, we review several studies that begin to examine the neural systems involved in incidental emotion regulation. Because these studies aim to identify when and where emotion regulatory processes occur, but without relying on explicit regulation instructions or self-reports that the emotion regulation has occurred, they also provide methodological tools that can be used to assess emotion regulation tendency.

\section{The Neuroscience of Incidental Emotion Regulation}

Studies on incidental emotion regulation can address the questions raised above at the same time that they help to broaden the theoretical horizons of traditional forms of emotion regulation. Incidental forms of emotion regulation have received relatively little empirical attention (cf. Mauss, Evers, Wilhelm, \& Gross, 2006); however, the neuroimaging tools now available remove some of the barriers that have until now prevented researchers from investigating incidental emotion regulation. 
What might studies investigating the neuroscience of incidental emotion regulation look like? At the most basic level, these studies should include a regulation condition, in which emotional stimuli are presented and affect is modulated without intent and/or outside of awareness, and a control condition, in which the same stimuli produce an affective response in the absence of incidental or intentional emotion regulation. Additionally, studies on incidental emotion regulation should complement the existing literature on intentional emotion regulation by addressing one or more of the open questions discussed above. Studies that broaden the operationalization of emotion regulation to new strategies, that isolate the key psychological processes involved in emotion regulation, or clarify how those processes operate in daily life would be particularly pertinent. Below, we review several studies that meet these criteria.

\section{Examples of research on incidental emotion regulation}

A number of existing studies begin to yield insights into a neuroscience of incidental emotion regulation, though it has not yet been studied under that name. Instead, researchers have noted that engaging in certain intentional activities can have incidental or unintended effects on affect-related processes (e.g. attention to or appraisal of emotional cues; reviewed by Phillips, Ladouceur, \& Drevets, 2008). Below, we review studies that most closely resemble intentional emotion regulation in their effects on affective response channels but operate outside of awareness or intention. Each study provides both empirical data relative to understanding incidental emotion regulation and methodological techniques that can be exploited in further investigations.

Affect labeling. 'Affect labeling' tasks (Hariri, Bookheimer, \& Mazziotta, 2000; Lieberman et al., 2007) are one technique for examining incidental emotion regulation. In these tasks, participants are presented with an emotion face target, and are instructed to identify the emotion depicted in the face by matching it to a similar emotional face ('match') or to a linguistic label for the emotion ('label'). In both cases, the comparison is made by selecting one of two options (either emotional faces or emotion words) that best corresponds to the target face (Figure 2). A task matching geometric shapes is used as a control condition. In both cases, participants are attending to affective features of the stimulus. The critical difference between them is whether the emotional information is represented visually or linguistically.

Results from these studies consistently find greater activation in right VLPFC during 'label' than 'match', and greater bilateral amygdala in 'match' than 'label'. In one study, connectivity analyses revealed greater inverse connectivity between the amygdala and right VLPFC during 'label' than 'match' (Hariri et al., 2000). Together, this pattern of finding suggests an 
(a)
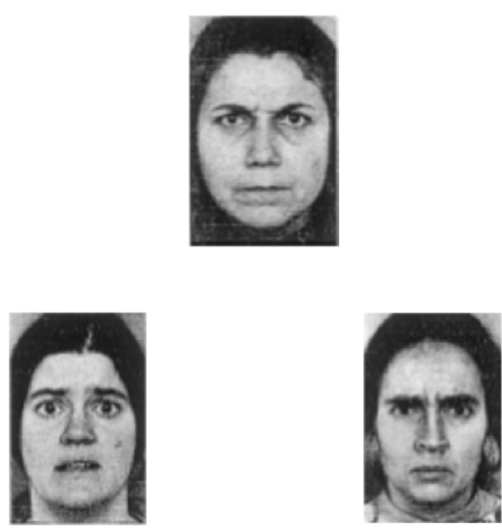

(b)

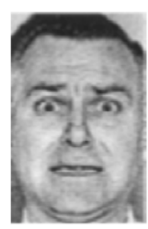

AFRAID

ANGRY

(c)
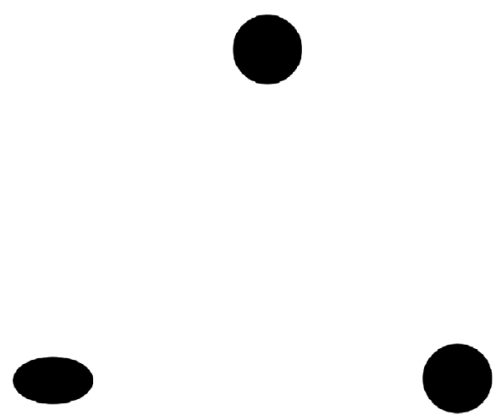

Figure 2 The 'affect labeling' task used by Hariri et al. (2000). In each case the participant pairs the target (top) with the more similar of the two options (bottom). (A) The affect 'match' condition where the comparison is based on visual similarity between the target and the pair. (B) The affect 'label' condition where the comparison is based on semantic similarity between the affect shown in the target and the linguistic labels. (C) The control condition based on matching of geometric shapes. Reprinted with permission. 
inhibitory relationship between amygdala and right VLPFC that is specifically engaged during the processing of linguistic affective information.

How is affect labeling a form of emotion regulation? It is not, according to conventional definitions of emotion regulation, because it lacks both the intention to reduce affective experience and the awareness that the reduction is occurring. However, although those differences qualify affect labeling as a distinct strategy phenomenologically, we suggest that it is similar to conscious emotion regulation in many ways. For example, labeling elicits similar reductions in later self-reported affect and physiological responding (Lieberman, Crockett, Inagaki, \& Tabibnia, forthcoming), and shares a similar (but not identical) pattern of neural activity with intentional emotion regulation. A critical question for the field moving forward is how to identify emotion regulation in the absence of intention or even awareness. We discuss several of the possible criteria in the section below entitled 'Criteria for regulation'.

Incidental regulation in service of other task goals. Tasks that require ignoring emotional distracters provide another way to study incidental emotion regulation. For example, Egner and colleagues (Egner, Etkins, Gale, \& Hirsch, 2008; Etkin, Enger, Peraza, Kandel, \& Hirsch, 2006) use a modified version of the emotional Stroop interference task to examine the neural systems associated with attention away from regulating emotional versus non-emotional distracters. In these tasks, participants respond by identifying the emotional expression on a target face that is overlayed with some potentially distracting information. The distracting information is either congruent or incongruent with the target face, and either emotional or non-emotional. For example, a non-emotional congruent distracter would be the word 'MALE' over a male fear face, and an emotional incongruent distracter would be the word 'FEAR' over a male happy face. Because the task requires responding based solely on the content of the target face, the overlay words are irrelevant. In the congruent conditions, the distracter words do not need to be ignored since a response based either on the distracters or the target will be correct. In contrast, participants must regulate their attention to the incongruent distracters to be able to complete the task. The authors found the rostral anterior cingulate cortex (rACC) was associated with regulation of attention to emotional (but not non-emotional) distracters, and that increases in activation in the rACC was coupled with decreases of activation in the amygdala on a trial-bytrial basis. Participants did not receive instructions to regulate their emotions, and thus the recruitment of the rACC, and the connectivity between the rACC and amygdala, were entirely incidental to the main task yet were presumably engaged to aid in completing the incongruent trials. A related study observed increased rACC activation in a pair-matching task when negative (but not neutral) faces were task-irrelevant, but not when the same faces were task-relevant (Vuilleumier, Armony, Driver, \& Dolan, 2001). Studies like these can examine how people engage in 
spontaneous regulation on an everyday basis, and identify the common and distinct neural systems involved in that type of regulation.

Contextual modulation of affective responses. Another type of incidental emotion regulation occurs when contextual factors alter an affective response outside of awareness. Even without self-reports of emotional experience, this effect can be observed using neuroimaging by identifying limbic system activity in one condition, and then a pattern of decreased activity in those same limbic structures coupled with increased prefrontal cortical activity in the other condition. Together, these findings would suggest that some process beyond simple disengagement from the affective stimuli is involved in the limbic reductions.

For example, Hare, Tottenham, Davidson, Glover, and Casey (2005) used an emotion go/no-go task to examine the effect of contextual information on neural responses to fear stimuli. In the task, participants responded to each of a series of fearful face stimuli by pressing a button ('go' trials). Those fearful 'go' faces were intermixed with occasional 'no-go' trials where participants were required to withhold a button press. Because the 'go' trials occur most of the time, a prepotent 'go' response builds in the subject that requires effort to overcome on the 'no-go' trials. The 'no-go' trials in this study were indicated by either neutral or happy face stimuli. The authors observed robust amygdala activation in response to the fearful faces in blocks when they were paired with neutral 'no-go' stimuli. However, in blocks when the fearful faces were paired with happy 'no-go' faces, the authors found a relative reduction in amygdala activity and an increase in caudate and VLPFC during the fear trials. There was also an increase in response time to the fearful trials in this condition. Here, a contextual factor - whether the occasional 'no-go' trials were neutral or happy altered the neural affective response to fearful face stimuli that were identical across the conditions. This study provides intriguing evidence that contextual factors can produce regulation-like effects in the absence of any intention to control one's emotions.

In another study demonstrating contextual effects producing incidental regulation, Kim et al. (2004) compared the neural response to identical pictures of surprise faces that were immediately preceded by either positive or negative information (e.g. 'She just won/lost $\$ 500$ '). In response to the same visual stimuli, the authors observed increased left amygdala activation during faces that were preceded by negative (relative to positive) information, and increased rACC activation during faces that were preceded by positive (relative to negative) information. In each of these studies, participants were not instructed to regulate their affective response, nor was their attention drawn to their own affective experience during the tasks. Nonetheless, subtle changes in the situational context such as the contextual information resulted in modulation of neural systems involved in affect, coupled with increases in prefrontal cortex activity. 
Individual differences in regulation tendencies. Another way of measuring incidental emotion regulation is to leverage individual differences in the tendency to spontaneously engage in emotion regulation, particularly in a context when there is no explicit instruction or awareness of emotion regulation. Jackson et al. (2003) capitalized on the high temporal resolution of a physiological measure of stimulus valence (startle eyeblink) to obtain an on-line measure of affective responses to emotional images. Using electroencephalography (EEG), they also derived a measure for each participant of resting hemispheric asymmetry between the right and left prefrontal cortices. They found that individuals with relatively higher baseline activation in the left DLPFC showed reduced affective responses (as indexed by startle magnitude) following the presentation of emotional images. Importantly, this reduction did not begin until 5-6 seconds following picture onset, suggesting that these individuals had an initial affective response that changed over the course of the stimulus presentation. The extent to which this difference reflects implicit emotion regulation or differences in temporal duration of emotional reactivity for another reason is unclear, but warrants further investigation. Regardless, and as in the previous studies, this change appears to have occurred spontaneously, with no explicit instruction, intention, or knowledge of the process.

\section{Theoretical considerations}

The review describes only a few of the many possible ways to address incidental emotional regulation using neuroscience approaches. Along with these methodological developments come substantial and interesting theoretical issues. Below, we briefly describe some of these and suggest ways of addressing them in the future.

Criteria for regulation. In each of the studies above, how are we to tell whether someone is incidentally regulating an emotion, or simply having a weaker initial affective response? More generally, how is one to conduct research on incidental emotion regulation in the absence of clear criteria for reduction in emotion? The studies above provide insights into these questions. First, it is critical to address the possibility that reductions or changes in affect are merely due to distraction or other differences in the task conditions not related to regulatory processes. This may necessitate additional control experiments to rule out these possibilities (e.g. Lieberman et al., 2007). Second, examining the time course of the affective measure can clarify whether affect indeed changes across time or is simply different between conditions (Jackson et al., 2003). For example, suppose a researcher wanted to compare the effectiveness of two incidental emotion regulation conditions, $\mathrm{A}$ and $\mathrm{B}$, in reducing affective responding (e.g. neural activation) in a 10-second time window. The researcher observes a comparable increase in conditions $\mathrm{A}$ and $\mathrm{B}$ from 0 to 3 seconds following 
the onset of the stimulus followed by a sustained increase in A but a diminished response in B from 4 seconds onward. The initial rise of responding in condition $\mathrm{B}$ followed by a decrease provides evidence for incidental emotion regulation in that condition that is less likely to be confounded with habituation or lack of attention to the stimulus. Finally, functional connectivity among regions might indicate active and dynamic neural engagement with the task that is unlikely to result from other confounding processes such as distraction or differential task difficulty (e.g. Hariri et al., 2000).

Though each of these techniques can be used to suggest the presence of regulation, one of the challenges for the field moving forward will be to reach consensus on what criteria are necessary and sufficient in order to conclude that emotion regulation has occurred. At this early point, we posit two criteria - one regarding the process and another regarding the outcome - for establishing that incidental emotion regulation occurred. First, some neuropsychological process that otherwise would not have occurred must take place. This process might be initiated by some unrelated cognitive task (as in affect labeling) or a situational cue that generates a spontaneous change in the appraisal of a stimulus. Second, there must be a change in affect relative to what would have occurred without the incidental process. Though affect has long eluded easy definition, there is consensus that self-reported experience is only one of many channels of affective responding. We suggest that central nervous system activity be counted among these as a complement to other physiological markers of affect such as peripheral nervous system responses. Additionally, behavioral indicators of affect such as facial expression, vocal tone and body posture can be used to corroborate changes in affect produced by incidental emotion regulation. Establishing change in several of these response channels is even more important to document that emotion regulation has occurred in the absence of self-reported change in affective experience.

Can incidental regulation be effortful? Although it is possible that some incidental emotion regulation will require little effort, other forms may be quite effortful while still being incidental. For example, a long and fruitful line of work by Pennebaker and colleagues (Pennebaker \& Beall, 1986; Pennebaker, 1997) has shown that expressive writing (compared to writing about trivial topics) reduces negative affect in addition to a host of longer-term physical health benefits (e.g. Stanton et al., 2002). Participants in these studies have no overt intention to regulate their emotions, though they are clearly engaging in an effortful task. Whether to classify these sorts of effortful but unintentional types of emotion regulation as 'incidental', intentional' or to create a new hybrid category, or whether category distinctions are useful at all, is a challenge for the field moving forward. For now, we suggest that comparing the neural systems involved in these various forms of emotion regulation is a useful tool in addressing this challenge. 
Instructed or spontaneous emotion regulation? Earlier we made the distinction between how a neurocognitive system might be made to function under specific instructions versus how it tends to function spontaneously. Most of the studies reviewed above on intentional emotion regulation are investigations of the capacity of the system to engage in a particular set of processes. The studies seek to understand the way the brain regulates responses to affective stimuli by carefully manipulating a known set of processes (e.g. reappraisal vs. suppression). This is a reasonable first step toward understanding the neural processes that correspond with intentional emotion regulation, and it might well be the case that many people spontaneously engage in intentional emotion regulation at least part of the time. Thus, it is possible that we already know quite a bit about the neural systems involved in spontaneous emotion regulation.

However, given the busy and cognitively demanding nature of the world, it seems likely that at least some spontaneous emotion regulation is incidental insofar as it occurs outside of awareness and without deliberate intent. A set of issues that remains to be resolved by emotion regulation researchers involves the degree to which spontaneous emotion regulation is intentional or incidental, for whom, to what degree, and under which conditions. Though these issues are not specific to neuroimaging investigations of emotion regulation, we believe that neuroimaging and other physiological measures will be particularly valuable in examining spontaneous emotion regulation because those techniques can provide an unobtrusive window into ongoing mental processes. We hope that a better understanding of the neural processes involved in intentional and incidental emotion regulation can help researchers better characterize the ways people engage in spontaneous emotion regulation.

\section{Conclusion}

At this point, it is premature to make many firm conclusions about the neural systems that are broadly involved in emotion modulation. Incidental emotion regulation is hardly understood, and it has yet to be compared directly to more intentional forms of emotion regulation. However, some patterns are beginning to emerge from the studies reviewed here suggesting that intentional and incidental emotion regulation recruit several overlapping and distinct regions. In terms of shared neural systems, both forms of emotion regulation recruit prefrontal structures, particularly the VLPFC, in down-regulating subcortical structures, particularly the amygdala. There is consensus that the PFC is involved in both forms of emotion modulation but exactly which parts depend on a variety of factors that include (to this point): whether language is involved in the task (and to what extent); whether the regulation is deliberate or incidental; and when during the stimulus presentation the neuroimaging is acquired. It also seems likely that the amygdala is only one of several limbic and subcortical 
structures that can be modulated, but appears disproportionately frequently because of its distinct anatomy and sensitivity to the particular stimuli that have been examined in emotion regulation studies.

Evidence for distinct neural systems is less consistent but with a few notable exceptions. The left ventrolateral and possibly dorsolateral PFC is present in several studies of intentional emotion regulation but almost never in studies of incidental emotion regulation. In contrast, the rACC appears consistently in studies of incidental but not intentional emotion regulation.

In addition to further clarifying these distinct and overlapping neural regions, we believe that an important direction for the field is to characterize the neural networks involved in emotion regulation and how the component regions interact. There is already some evidence of an inhibitory relationship between prefrontal and limbic structures, particularly between the amygdala and the ventrolateral and ventromedial PFC, but this network is only beginning to be explored. We are optimistic that the field will make fast strides in the coming years in addressing each of these open questions.

\section{Short Biography}

Matthew Lieberman's primary research interests are in the field of social cognitive neuroscience, which integrates concepts and methodologies from social psychology and cognitive neuroscience. He was recently honored with an APA early career award in recognition of his pioneering work in this field. Lieberman's previous publications include work on the neural bases of controlled and automatic processes, self-knowledge, interpersonal rejection, and cognitive dissonance. He is also the founding editor of the journal Social Cognitive and Affective Neuroscience (SCAN). Lieberman holds a BA in Philosophy and Psychology from Rutgers University and a PhD in Social Psychology from Harvard University. Currently, Lieberman is an Associate Professor in the Psychology Department at the University of California, Los Angeles.

Elliot Berkman is currently a doctoral candidate and National Research Service Award fellow in the Psychology Department at the University of California, Los Angeles. His research is aimed at understanding how humans succeed or fail in their long-term goals (e.g. smoking cessation). As part of this work, he examines basic goal processes such as self-regulation, motivation, and emotion, using a variety of tools including neuroimaging, laboratory studies, and longitudinal experience sampling. Berkman holds a BA in Psychology and a BS in Mathematics from Stanford University and MAs in Social Psychology from Stanford and UCLA.

\section{Endnote}

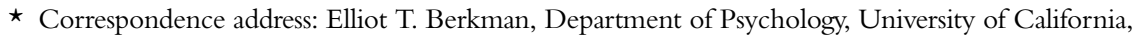
Los Angeles, 1285 Franz Hall, Box 951563, Los Angeles, CA 90095-1563, USA. E-mail: berkman@ucla.edu 


\section{References}

Banks, S. J., Eddy, K. T., Angstadt, M., Nathan, P. J., \& Phan, K. L. (2007). Amygdala-frontal connectivity during emotion regulation. Social Cognitive and Affective Neuroscience, 2, 303-312.

Beauregard, M., Levesque, J., \& Bourgouin, P. (2001). Neural correlates of conscious selfregulation of emotion. Journal of Neuroscience, 21, 6993-7000.

Blakemore, S. J., Rees, G., \& Frith, C. D. (1998). How do we predict the consequences of our actions? A functional imaging study. Neuropsychologia, 36, 521-529.

Casey, B. J., Cohen, J. D., O’Craven, K., Davidson, R. J., Irwin, W., Nelson, C. A., et al. (1998). Reproducibility of fMRI results across four institutions using a spatial working memory task. Neuroimage, 8, 249-261.

Eippert, F., Veit, R., Weiskopf, N., Erb, M., Birbaumer, N., \& Anders, S. (2007). Regulation of emotional responses elicited by threat-related stimuli. Human Brain Mapping, 28, 409-423.

Eisenberger, N. I., Lieberman, M. D., \& Williams, K. D. (2003). Does rejection hurt? An FMRI study of social exclusion. Science, 302, 290-292.

Ekman, P. (1992). An argument for basic emotions. Cognition and Emotion, 6 (3/4), 169-200.

Enger, T., Etkins, A., Gale, S., \& Hirsch, J. (2008). Dissociable neural systems resolve conflict from emotional versus nonemotional distracters. Cereberal Cortex, 18, 1475-1484.

Etkin, A., Enger, T., Peraza, D. M., Kandel, E. R., \& Hirsch, J. (2006). Resolving emotional confilict: A role for the rostral anterior cingulated cortex in modulating activity in the amygdala. Neuron, 51, 871-882.

Goldin, P. R., McRae, K., Ramel, W., \& Gross, J. J. (2008). The neural bases of emotion regulation: Reappraisal and suppression of negative emotion. Biological Psychiatry, 63, 577-586.

Gross, J. J. (1998a). Antecedent- and response-focused emotion regulation: Divergent consequences for experience, expression, and physiology. Journal of Personality \& Social Psychology, 74, 224-237.

Gross, J. J. (1998b). The emerging field of emotion regulation: An integrative review. Review of General Psychology, 2, 271-299.

Gross, J. J. (1999). Emotion regulation: Past, present, future. Cognition \& Emotion, 13, 551-573. Gross, J. J. (Ed.). (2007). Handbook of Emotion Regulation. New York, NY: The Guilford Press. Gross, J. J., \& Thompson, R. A. (2007). Emotion regulation: Conceptual foundations. In J. J. Gross (Ed.), Handbook of Emotion Regulation (pp. 3-24). New York, NY: The Guilford Press.

Gross, J. J., Richards, J. M., \& John, O. P. (2006). Emotion regulation in everyday life. In D. K. Snyder, J. A. Simpson \& J. N. Hughes (Eds.), Emotion Regulation in Families: Pathways to Dysfunction and Health (pp. 13-35). Washington, DC: American Psychological Association.

Hare, T. A., Tottenham, N., Davidson, M. C., Glover, G. H., \& Casey, B. J. (2005). Contributions of amygdala and striatal activity in emotion regulation. Biological Psychiatry, 57 (6), 624-632.

Harenski, C. L., \& Hamann, S. (2006). Neural correlates of regulating negative emotions related to moral violations. Neuroimage, 30, 313-24.

Hariri, A. R., Bookheimer, S. Y., \& Mazziotta, J. C. (2000). Modulating emotional responses: Effects of a neocortical network on the limbic system. Neuroreport: For Rapid Communication of Neuroscience Research, 11, 43-48.

Herwig, U., Baumgartner, T., Kaffenberger, T., Bruhl, A., Kottlow, M., Schreiter-Gasser, U., et al. (2007). Modulation of anticipatory emotion and perception processing by cognitive control. Neuroimage, 37, 652-662.

Jackson, D. C., Mueller, C. J., Dolski, I., Dalton, K. M., Nitschke, J. B., Urry, H. L., et al. (2003). Now you feel it, now you don't: Frontal brain electrical asymmetry and individual differences in emotion regulation. Psychological Science, 14, 612-617.

Kalisch, R., Wiech, K., Critchley, H. D., Seymour, B., O’Doherty, J. P., Oakley, D. A., et al. (2005). Anxiety reduction through detachment: subjective, physiological, and neural effects. Journal of Cognitive Neuroscience, 17, 874-883.

Kalisch, R., Wiech, K., Herrmann, K., \& Dolan, R. J. (2006). Neural correlates of selfdistraction from anxiety and a process model of cognitive emotion regulation. Journal of Cognitive Neuroscience, 18, 1266-1276.

Kim, H., Somerville, L. H., Johnstone, T., Polis, S., Alexander, A. L., Shin, L. M., et al. (2004). Contextual modulation of amygdala responsivity to surprised faces Journal of Cognitive Neuroscience, 16, 1730-1745. 
Kim, S. H., \& Hamann, S. (2007). Neural correlates of positive and negative emotion regulation. Journal of Cognitive Neuroscience, 19, 776-798.

Kjaer, T. W., Nowak, M., Kjaer, K. W., Lou, A. R., \& Lou, H. C. (2001). Precuneus-prefrontal activity during awareness of visual verbal stimuli. Consciousness \& Cognition, 10, 356-365.

Lee, T-W., Dolan, R. J., \& Critchley, H. D. (2008). Controlling emotional expression: behavioral and neural correlates of nonimitative emotional responses. Cerebral Cortex, 18, 104-113.

Levesque, J., Eugene, F., Joanette, Y., Paquette, V., Mensour, B., Beaudoin, G., et al. (2003). Neural circuitry underlying voluntary suppression of sadness. Biological Psychiatry, 53, 502-510.

Lieberman, M. D., Crockett, M. J., Inagaki, T., \& Tabibnia, G. (forthcoming). Affect labeling diminishes affective responses: Evidence from self-report and physiology.

Lieberman, M. D., Eisenberger, N. I., Crockett, M. J., Tom, S. M., Pfeifer, J. H., \& Way, B. M. (2007). Putting feelings into words: Affect labeling disrupts amygdala activity in response to affective stimuli. Psychological Science, 18, 421-428.

Mauss, I. B., Evers, C., Wilhelm, F. H., \& Gross, J. J. (2006). How to bite your tongue without blowing your top: Implicit evaluation of emotion regulation predicts affective responding to anger provocation. Personality and Social Psychology Bulletin, 32, 589-602.

McRae, K., Ochsner, K. N., Mauss, I. B., Gabrieli, J. J. D., \& Gross, J. J. (2008). Gender differences in emotion regulation: An fMRI Study of cognitive reappraisal. Group Processes $\&$ Intergroup Relations, 11, 143-162.

Ochsner, K. N., Bunge, S. A., Gross, J. J., \& Gabrieli, J. J. D. (2002). Rethinking feelings: An fMRI study of the cognitive regulation of emotion. Journal of Cognitive Neuroscience, 14, $1215-1229$.

Ochsner, K. N., Ray, R. D., Cooper, J. C., Robertson, E. R., Chopra, S., Gabrieli, J. D., et al. (2004). For better or for worse: neural systems supporting the cognitive down- and upregulation of negative emotion. Neuroimage, 23, 483-499.

Ohira, H., Nomura, M., Ichikawa, N., Isowa, T., Iidaka, T., Sato, A., et al. (2006). Association of neural and physiological responses during voluntary emotion suppression. Neuroimage, 29, 721-733.

Pennebaker, J. W., \& Beall, S. K. (1986). Confronting a traumatic event. Toward an understanding of inhibition and disease. Journal of Abnormal Psychology, 95, 274-281.

Pennebaker, J. W. (1997). Writing about emotional experiences as a therapeutic process. Psychological Science, 8, 162-166.

Phan, K. L., Fitzgerald, D. A., Nathan, P. J., Moore, G. J., Uhde, T. W., \& Tancer, M. E. (2005). Neural substrates for voluntary suppression of negative affect: A functional magnetic resonance imaging study. Biological Psychiatry, 57, 210-219.

Phillips, M. L., Ladouceur, C. D., \& Drevets, W. C. (2008). A neural model of voluntary and automatic emotion regulation: Implications for understanding the pathphysiology and neuro development of bipolar disorder. Molecular Psychiatry, 13 (9), 833-857.

Poldrack, R. A. (2006). Can cognitive processes be inferred from neuroimaging data? Trends in Cognitive Sciences, 10, 59-63.

Schaefer, A., Collette, F., Philippot, P., Van der Linden, M., Laureys, S., Delfiore, G., et al. (2003). Neural correlates of 'hot' and 'cold' emotional processing: a multilevel approach to the functional anatomy of emotion. Neuroimage, 18, 938-949.

Stanton, A. L., Danoff-Burg, S., Sworowski, L. A., Collins, C. A., Branstetter, A. D., Rodriguez-Hanley, A., et al. (2002). Randomized, controlled trial of written emotional expression and benefit finding in breast cancer patients. Journal of Clinical Oncology, 15, 4160-4168.

Tabibnia, G., Satpute, A. B., \& Lieberman, M. D. (2008). The sunny side of fairness: Fairness preference activates reward circuitry (and disregarding unfairness activates self-control circuitry). Psychological Science, 19, 339-347.

Urry, H. L., van Reekum, C. M., Johnstone, T., Kalin, N. H., Thurow, M. E., Schaefer, H. S., et al. (2006). Amygdala and ventromedial prefrontal cortex are inversely coupled during regulation of negative affect and predict the diurnal pattern of cortisol secretion among older adults. Journal of Neuroscience, 26, 4415-4425.

Vuilleumier, P., Armony, J. L., Driver, J., \& Dolan, R. J. (2001). Effects of attention and emotion on face processing in the human brain: an event-related fMRI study. Neuron, 30, 829-841.

Wager, T. D., Davidson, M. L., Hughes, B. L., Lindquist, M. A., \& Ochsner, K. N. (2008). Prefrontal-subcortical pathways mediating successful emotion regulation. Neuron, 59, 1037-1050. 